\title{
Structural Properties of Liquid SiC during Rapid Solidification
}

\author{
WanJun Yan, ${ }^{1,2}$ TingHong Gao, ${ }^{1}$ XiaoTian Guo, ${ }^{1,2}$ YunXiang Qin, ${ }^{1}$ and Quan Xie \\ ${ }^{1}$ Institute of New Type Optoelectronic Materials and Technology, School of Electronic Information, \\ Guizhou University, Guiyang 550025, China \\ ${ }^{2}$ College of Electronic and Information Engineering, Anshun University, Anshun 561000, China
}

Correspondence should be addressed to Quan Xie; qxie@gzu.edu.cn

Received 6 August 2013; Accepted 17 September 2013

Academic Editors: A. F. Borghesani and A. Kochur

Copyright (C) 2013 WanJun Yan et al. This is an open access article distributed under the Creative Commons Attribution License, which permits unrestricted use, distribution, and reproduction in any medium, provided the original work is properly cited.

\begin{abstract}
The rapid solidification of liquid silicon carbide $(\mathrm{SiC})$ is studied by molecular dynamic simulation using the Tersoff potential. The structural properties of liquid and amorphous $\mathrm{SiC}$ are analyzed by the radial distribution function, angular distribution function, coordination number, and visualization technology. Results show that both heteronuclear and homonuclear bonds exist and no atomic segregation occurs during solidification. The bond angles of silicon and carbon atoms are distributed at around $109^{\circ}$ and $120^{\circ}$, respectively, and the average coordination number is $<4$. Threefold carbon atoms and fourfold silicon atoms are linked together by six typical structures and ultimately form a random network of amorphous structure. The simulated results help understand the structural properties of liquid and amorphous $\mathrm{SiC}$, as well as other similar semiconductor alloys.
\end{abstract}

\section{Introduction}

Interest in the properties of disordered materials is continuously growing because of their technological applications and the fundamental questions related to them. Researching the transition process of liquid to solid and the microstructure evolution law during rapid solidification are research hotspots in condensed matter physics and material science [1]. The atomic arrangement and formation processes of microstructure cannot be directly observed during rapid solidification, but detailed structural information at the atomic scale can be obtained by molecular dynamics (MD) simulations. MD simulation is widely used to study the crystallization or amorphization of liquid metals and alloys [2-5]. By contrast, studies on semiconductor alloys are limited.

Silicon carbide $(\mathrm{SiC})$ is a wide-band-gap semiconductor with excellent chemical stability, electronic properties, high rigidity, and high hardness [6]. Considering that the macroscopic properties mainly depend on the SiC microstructure, a clear picture of atom packing during formation processes is important. MD simulation has been successfully used to research the mechanical properties [7], surface $[8,9]$, defect [10], crystal growth [11], and nanotubes or nanowires [1215] of SiC. No theoretical study has been conducted on the structural properties of liquid $\mathrm{SiC}$ during solidification.
In this paper, we simulate solidification of $3 \mathrm{C}-\mathrm{SiC}$ by MD using the Tersoff potential. The structural properties are analyzed in detail by the radial distribution function (RDF), angular distribution function (ADF), coordination number $(\mathrm{CN})$, and visualization technology. This work is expected to present images of the structural properties of liquid SiC ( $l$ $\mathrm{SiC}$ ) and amorphous $\mathrm{SiC}(a-\mathrm{SiC})$ during rapid solidification, thereby providing insight into other similar semiconductor alloys.

\section{Simulation Details and Analysis Methods}

In the simulation study, $4000 \mathrm{Si}$ atoms and $4000 \mathrm{C}$ atoms are initially placed on the diamond lattice sites in a cubic MD cell with periodic boundary conditions. The size of the MD cell is $10 a_{0} \times 10 a_{0} \times 10 a_{0}$, where $a_{0}$ is the equilibrium lattice parameter of $\operatorname{SiC}\left(a_{0}=4.43 \AA\right)$. The Newtonian equations of motion are integrated using the velocity Verlet algorithm [16] with time step $\Delta t=1 \mathrm{fs}\left(1 \times 10^{-15} \mathrm{~s}\right)$. The atomic interactions are described using the Tersoff potential [17], which is known to simulate the covalent system with its complex structure and energy. The Tersoff potential is also widely used for various applications of silicon, carbon, germanium, and their compounds. 


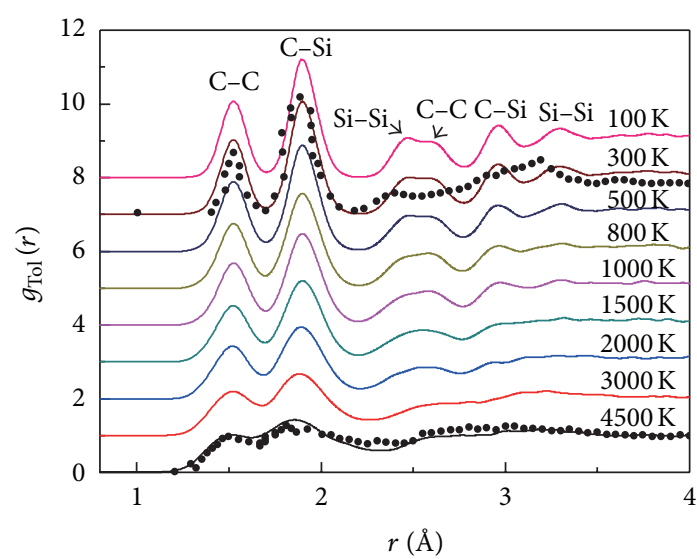

- P. C. Kelires MC

- Our MD simulation

(a)

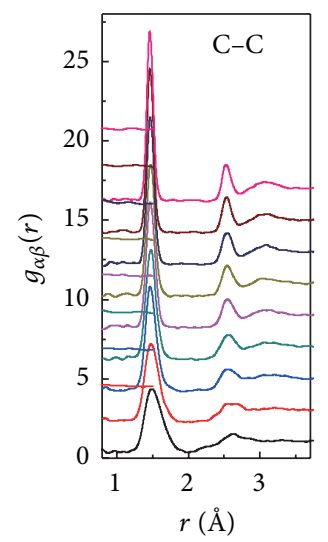

(b)
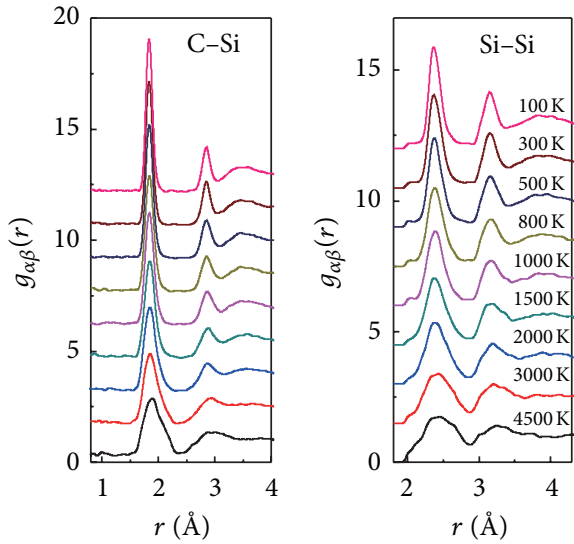

(c) (d)

FIGURE 1: The evolution of the RDF with decreased temperature. ((a) $g_{\mathrm{Tol}}(r)$; (b) $g_{\mathrm{C}-\mathrm{C}}(r)$; (c) $g_{\mathrm{C}-\mathrm{Si}}(r)$; (d) $\left.g_{\mathrm{Si}-\mathrm{Si}}(r)\right)$.

To generate the liquid configuration, the $\mathrm{SiC}$ crystal is heated from $100 \mathrm{~K}$ to $4500 \mathrm{~K}$, with isothermal running for $40 \mathrm{ps}$ at $4500 \mathrm{~K}$ to achieve an equilibrium liquid state. Then, the temperature gradually decreases to $100 \mathrm{~K}$ at a cooling rate of $1.0 \times 10^{10} \mathrm{~K} / \mathrm{s}$. The configurations are recorded every $100 \mathrm{~K}$ during solidification. The structural properties of $l$-SiC and $a-\mathrm{SiC}$ are analyzed by $\mathrm{RDF}, \mathrm{ADF}, \mathrm{CN}$, and visualization technology.

\section{Results and Discussion}

3.1. Radial Distribution Function (RDF). The RDF is an important method of describing the structural ordering of condensed matter systems. The Fourier transform of RDF determines the corresponding partial static structure factor obtained by X-ray imaging; thus, the RDF is an important structural parameter for comparing experimental and theoretical results.

Figure 1 shows the evolution of the RDF with temperature decrease. Figure 1 shows that both heteronuclear and homonuclear bonds exist in the system. Figure 1(a) compares the results with those obtained by Kelires [18] using the Monte Carlo method (black solid round dot) at 4500 and $300 \mathrm{~K}$. The first and second peaks agree well at high and low temperatures, whereas a small difference can be seen from $2.4 \AA$ to $3.2 \AA$. Figures 1(b), 1(c), and 1(d) show that with decreased temperature, the first peaks of $\mathrm{C}-\mathrm{C}, \mathrm{C}-\mathrm{Si}$, and $\mathrm{Si}-\mathrm{Si}$ all become heightened and sharp, which indicates the uniformity of the bond length and the increase in shortrange order (SRO) for all kinds of bonds. The bond length of the $\mathrm{C}-\mathrm{C}$ bond gradually decreases from $1.5 \AA$ to $1.45 \AA$ with decreased temperature from $4500 \mathrm{~K}$ to $100 \mathrm{~K}$, and the bond length is close to that of graphite $(1.46 \AA)$. At $100 \mathrm{~K}$, the second peak of $g_{\mathrm{C}-\mathrm{C}}(r)$ is located at $\sim 2.55 \AA$, which corresponds to the distance of the $\mathrm{C}-\mathrm{C}$ bond in diamond. In other words, some $\mathrm{C}$ atoms form $\mathrm{C}-\mathrm{C}-\mathrm{C}$ SRO during solidification. The bond length of C-Si remains $1.85 \AA$ throughout solidification, which is very close to the results of Kaloyeros et al. [19] and Pascarelli et al. [20] as measured by extended X-ray absorption fine structure. The bond length of the Si-Si bond gradually decreases from $2.5 \AA$ to $2.35 \AA$ with temperature decrease. At $100 \mathrm{~K}$, the bond length of $\mathrm{Si}-\mathrm{Si}$ is close to that of the crystal Si at $2.36 \AA$, which indicates that some $\mathrm{Si}$ atoms form $\mathrm{Si}-\mathrm{Si}-\mathrm{Si}$ SRO.

3.2. Coordination Number $(\mathrm{CN})$. The $\mathrm{CN}$ is the average number of nearest-neighboring atoms used to describe the degree of tightness of atomic arrangement. For a multicomponent system, such a number must be separately defined for each type of atom pair. Thus, the partial coordination is indicated by the areas under the first peak (up to the minimum) in the partial RDF (PRDF) $g_{\alpha \beta}(r)$. Figure 2 shows the average CN of $\mathrm{C}$ and $\mathrm{Si}$ atoms versus the temperature.

Figure 2 shows that the average $\mathrm{CN}$ of carbon or silicon atoms is $<4$ at $4500 \mathrm{~K}$. The $\mathrm{CN}$ of Si and C increases with decreased temperature. At $<1500 \mathrm{~K}$, the $\mathrm{CNs}$ of Si and C are stable at 4.01 and 3.72, respectively. The change in the $\mathrm{CN}$ of silicon and carbon atoms during solidification is small, and the average $\mathrm{CN}$ of $\mathrm{SiC}$ is 3.87 at the end of solidification, which is close to the experiment results 3.99 [20] and 3.94 [21]. In liquid $\mathrm{SiC}$, neither the high-density phase of silicon (the CNs are 5.8-6.0 for pure liquid silicon [19] and 6.0 [18] for silicon in liquid SiGe) nor the liquid-liquid phase transformation occurs during liquid silicon solidification [21]. The CN of pure liquid carbon is 2.8-2.9 [19], which is lower than that of $\mathrm{SiC}$.

Table 1 shows the positions of the first three peaks of the $\mathrm{RDF}$ and the average of corresponding partial CNs at $100 \mathrm{~K}$.

In Table 1, 1.68 silicon and 2.33 carbon atoms on average are bonded to each silicon atom, that is, $z_{\mathrm{Si}-\mathrm{Si}}=1.68$ and $z_{\mathrm{Si}-\mathrm{C}}=2.33$, whereas 1.39 carbon and 2.33 silicon atoms are bonded to each carbon atom, that is, $z_{\mathrm{C}-\mathrm{C}}=1.39$ and $z_{\mathrm{C}-\mathrm{Si}}=2.33$. In total, silicon has 4.01 nearest neighbors $\left(z_{\mathrm{Si}}=\right.$ $4.01)$ and carbon has 3.72 nearest neighbors $\left(z_{\mathrm{C}}=3.72\right)$. 


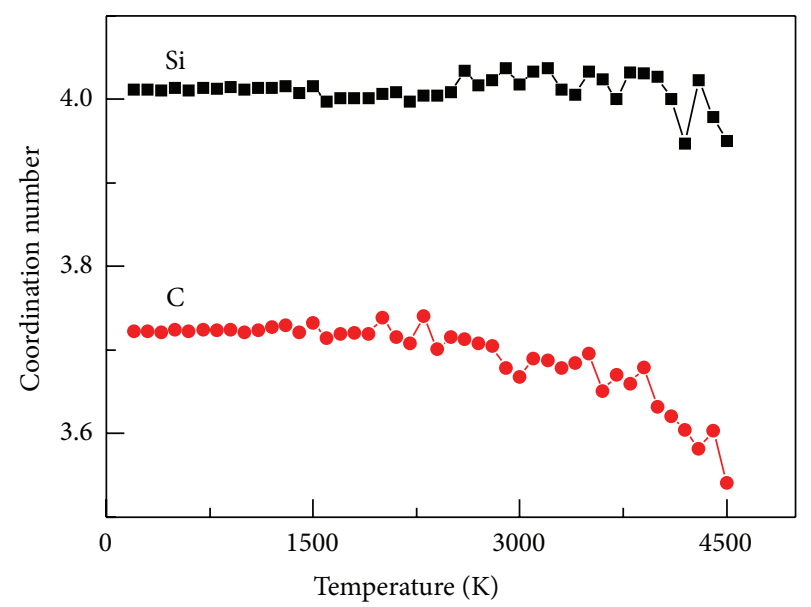

Figure 2: The average $\mathrm{CN}$ of $\mathrm{Si}$ and $\mathrm{C}$ with decreased temperature.

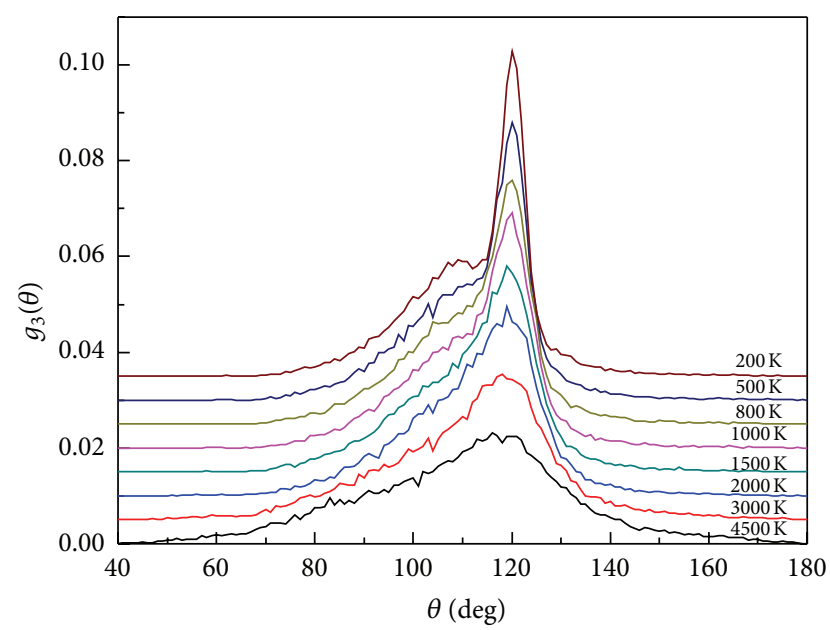

FIGURE 3: The evolution of the ADF with decreased temperature.

The final structure of quenched $\mathrm{SiC}$ cannot be described as a tetrahedral network because most carbon atoms are threefold coordinated.

3.3. Angular Distribution Function (ADF). The ADF can be used to describe the statistical average of angles formed with neighboring atoms. Figure 3 shows the relationship between the ADF and temperature. Figure 3 shows that the maximum peak of the ADF is located at $120^{\circ}$, which indicates that many threefold coordinated atoms exist in the system. Angular distribution near $109^{\circ}$ indicates some nonstandard tetrahedron units or other complex local structures. With decreased temperature, the range of the ADF narrows and the maximum peak gradually increases, which indicates that the atomic structure becomes more orderly. To reveal the transformation of the ADF, the ADF of silicon and carbon as center atoms are analyzed in detail. Figure 4 shows the ADF of silicon and carbon as center atoms at 4500 and $100 \mathrm{~K}$.

Figures 4(a) and 4(b) show that at $4500 \mathrm{~K}$, complex bond types exist in liquid SiC. Silicon atoms mainly form threefold, fourfold, fivefold, and sixfold coordination structures, and
TABLE 1: Position of the first three peaks of RDF and the average of corresponding partial $\mathrm{CN} z$ at $100 \mathrm{~K}$.

\begin{tabular}{lcccc}
\hline & $r_{1} / \AA$ & $r_{2} / \AA$ & $r_{3} / \AA$ & $z$ \\
\hline $\mathrm{C}-\mathrm{C}$ & $1.45(1.46)^{\mathrm{a}}$ & $2.55(2.52)^{\mathrm{b}}$ & $3.05(3.07)^{\mathrm{c}}$ & $1.39(1.19)^{\mathrm{e}}$ \\
$\mathrm{C}-\mathrm{Si}$ & $1.85(1.88)^{\mathrm{c}}$ & 2.85 & $3.40(3.60)^{\mathrm{c}}$ & $2.33(2.27)^{\mathrm{e}}$ \\
$\mathrm{Si}-\mathrm{Si}$ & $2.35(2.36)^{\mathrm{d}}$ & $3.15(3.07)^{\mathrm{c}}$ & $3.90(3.84)^{\mathrm{d}}$ & $1.68(1.75)^{\mathrm{e}}$ \\
\hline
\end{tabular}

${ }^{\mathrm{a}}$ Graphite, ${ }^{\mathrm{b}}$ diamond, ${ }^{\mathrm{c}} \mathrm{SiC}$ crystal, ${ }^{\mathrm{d}} \mathrm{Si}$ crystal, and ${ }^{\mathrm{e}}$ Kelires [18].

fourfold silicon atoms account for the greatest proportion. The bond angles of fourfold, fivefold, and threefold silicon atoms are distributed within the ranges of $96^{\circ}-109^{\circ}, \sim 90^{\circ}$, and $92^{\circ}-128^{\circ}$, respectively. Carbon atoms mainly form threefold, fourfold, and a small number of twofold coordination structures. The bond angles of threefold and fourfold carbon atoms are distributed within the ranges of $115^{\circ}-121^{\circ}$ and $97^{\circ}-121^{\circ}$, respectively. Figures $4(\mathrm{c})$ and $4(\mathrm{~d})$ show that silicon atoms mainly form fourfold coordination structures at $100 \mathrm{~K}$, and the main peak is located at $\sim 109^{\circ}$ (bond angle of tetrahedral). Carbon atoms mainly form a large number of threefold coordination structures, and the main peak is located at $\sim 120^{\circ}$ (bond angle of graphite). During solidification, silicon atoms tend to form fourfold coordination structures with a tetrahedral angle, whereas carbon atoms tend to form threefold coordination structures with a graphite angle. However, no graphite structure forms at the end of solidification, as discussed in the following section.

3.4. Microstructure Visualization Analysis. To present a clear image of microstructures during solidification, Figure 5 shows a change in the main structures of silicon and carbon as the center atoms with decreased temperature. We use $\mathrm{A} m \mathrm{~B} n$ to denote the structure. A is the center atom type and $m$ is the number of A. Meanwhile, B is the atom type that differs from $\mathrm{A}$ and $n$ is the number of $\mathrm{B}$. For example, C3Sil represents the carbon as the center atom, and the structure comprises three carbon atoms and one silicon atom. The green ball represents carbon atom, and the purple one represents silicon atom.

When carbon is the center atom, four kinds of threefold coordination structures exist, namely, C1Si3, C2Si2, C3Sil, and C4. Figures 5(a) and 5(b) show that the number of the four kinds of structures from more to less is $\mathrm{C} 2 \mathrm{Si} 2$, C3Sil, C1Si3, and C4. C4 has the least number of structures that indicates the absence of carbon atomic segregation throughout the entire solidification process. The number of the four kinds of structures during oscillation increases with decreased temperature from $4500 \mathrm{~K}$ to $1500 \mathrm{~K}$ and gradually tends to be stable when the temperature is $<1500 \mathrm{~K}$. When silicon is the center atom, five kinds of fourfold coordination structures exist: SilC4, Si2C3, Si3C2, Si4C1, and Si5. Figures 5(c) and 5(d) show that the number of the five kinds of structures from more to less is $\mathrm{Si} 3 \mathrm{C} 2, \mathrm{Si} 4 \mathrm{C} 1, \mathrm{Si} 2 \mathrm{C} 3, \mathrm{Si} 5$, and SilC4. Very few Si5 and SilC4 structures exist, indicating that no silicon atomic segregation occurs and no crystal structure forms throughout the entire solidification process.

In consideration of the threefold carbon atoms and fourfold silicon atoms accounting for a large proportion after solidification, the combination mode for these atoms must 


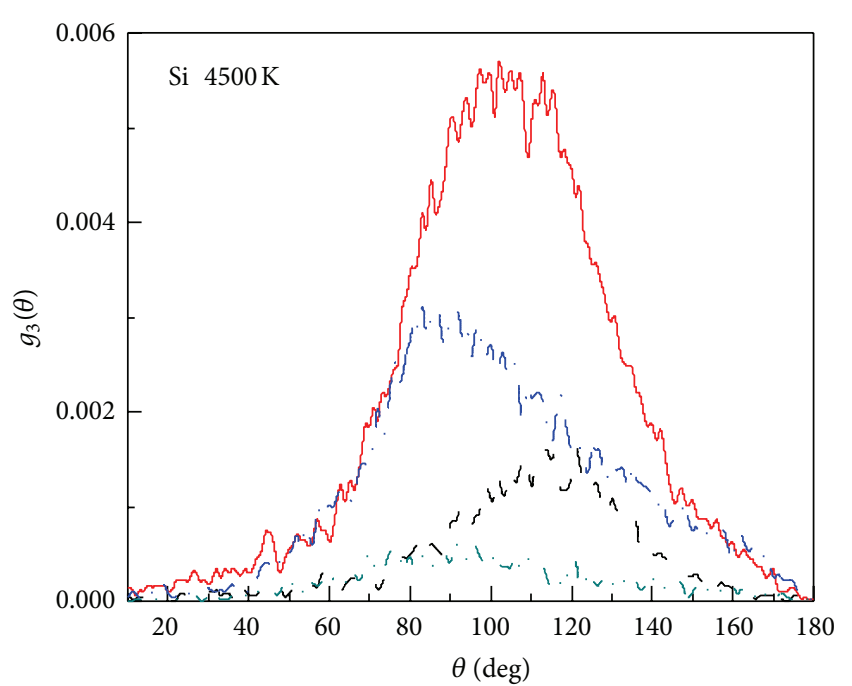

(a)

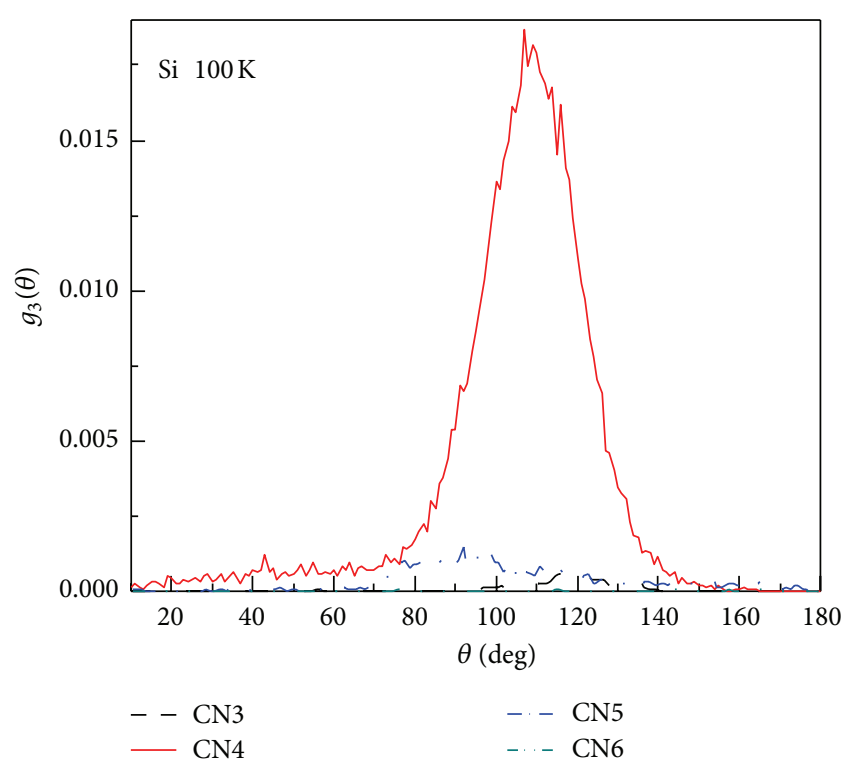

(c)

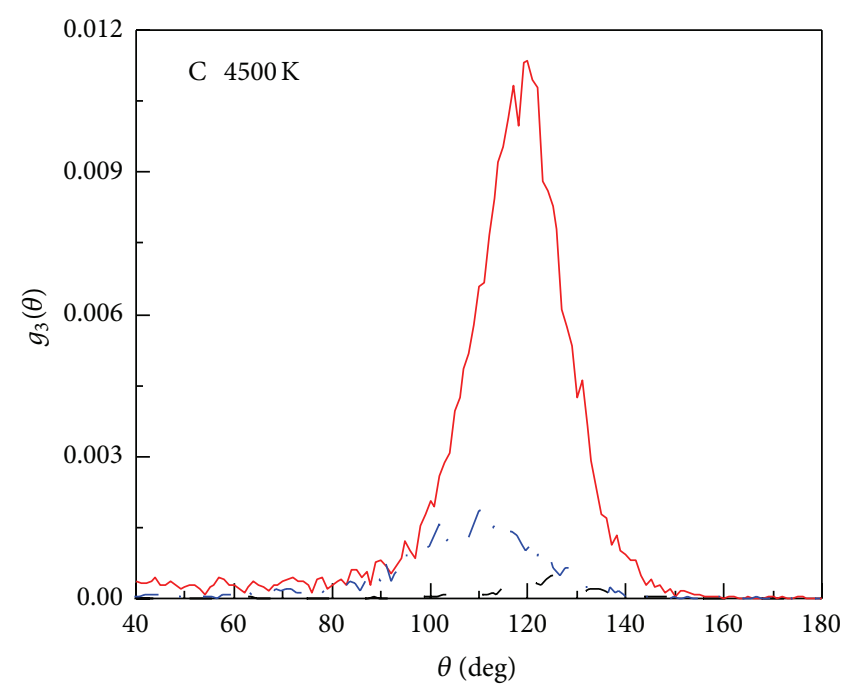

(b)

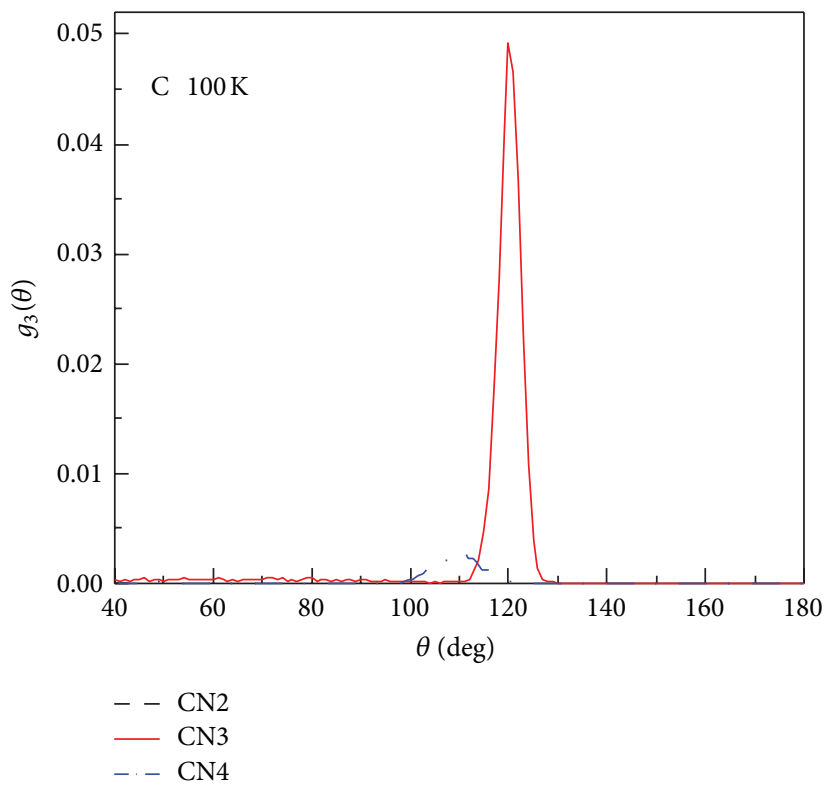

(d)

Figure 4: The ADF of $\mathrm{SiC}$ at $4500 \mathrm{~K}$ and $100 \mathrm{~K}$. ((a), (c) at $4500 \mathrm{~K}$ and $100 \mathrm{~K}, \mathrm{Si}$ as central atom, CN-3, 4, 5, 6; (b), (d) at $4500 \mathrm{~K}$ and $100 \mathrm{~K}, \mathrm{C}$ as central atom, $\mathrm{CN}-2,3,4)$.

be intensively investigated. Figure 6 shows the six typical combination modes of threefold carbon atoms and fourfold silicon atoms.

Figures 6(a), 6(b), and 6(c) represent the central atom as the neighboring atom of the other structure (the red bond connects two center atoms). Figure 6(a) shows two threefold coordination structures connected by two carbon atoms that are the center atoms of the two structures, and the two structures are not in the same plane. The bond angles of $\mathrm{Si}-\mathrm{C}-\mathrm{Si}$ and $\mathrm{C}-\mathrm{C}-\mathrm{Si}$ are both $\sim 120^{\circ}$, and the lengths of the $\mathrm{C}-\mathrm{C}$ and $\mathrm{C}-\mathrm{Si}$ bonds are $\sim 1.45$ and $\sim 1.85 \AA$, respectively, which corresponds to the location of the first peaks of $g_{\mathrm{C}-\mathrm{C}}(r)$ and $g_{\mathrm{C}-\mathrm{Si}}(r)$ in Figures 1(b) and 1(c). Figure 6(b) shows a threefold and a fourfold coordination structure connected by a carbon atom and a silicon atom, which are the center atoms of the two structures. Figure 6(c) shows two fourfold coordination structures connected by two silicon atoms, which are the center atoms of the two structures. Figures 6(d), 6(e), and 6(f) show the connection of the two structures by sharing a neighboring atom. Figure 6(d) shows two threefold coordination structures connected by sharing a neighbor carbon atom and forming a short-range structure $\mathrm{C}-\mathrm{C}-\mathrm{C}$. The distance of the two carbon atoms farthermost apart is $\sim 2.55 \AA$, which corresponds to the location of the second peak of $g_{\mathrm{C}-\mathrm{C}}(r)$ in Figure 1(b). Two structures form a twisty chain structure. Figure 6(e) shows a threefold and a fourfold coordination structure connected by sharing the neighboring carbon atom. Figure 6(f) shows two fourfold coordination 


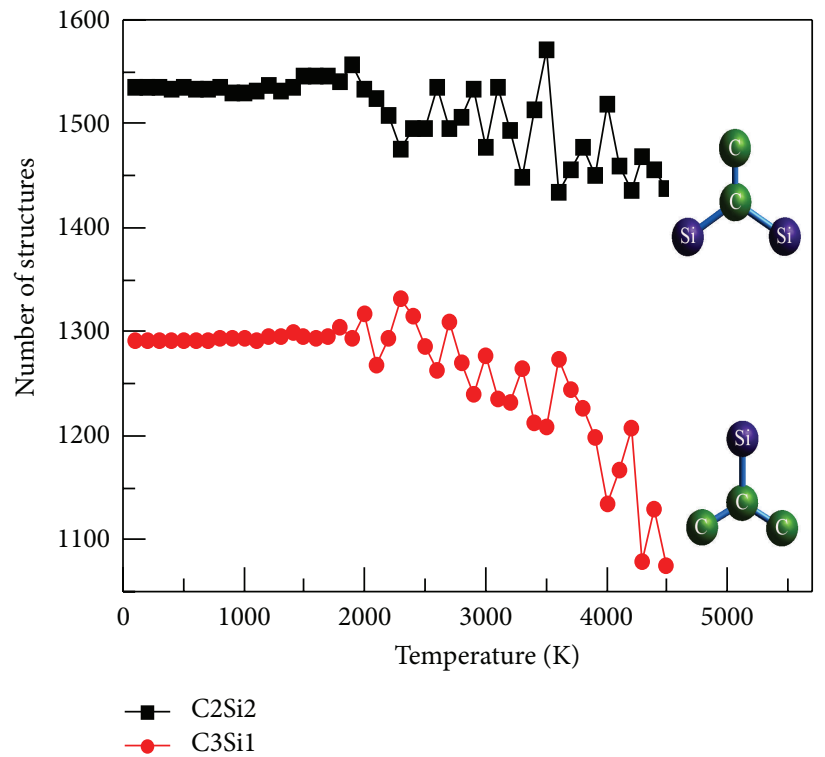

(a)

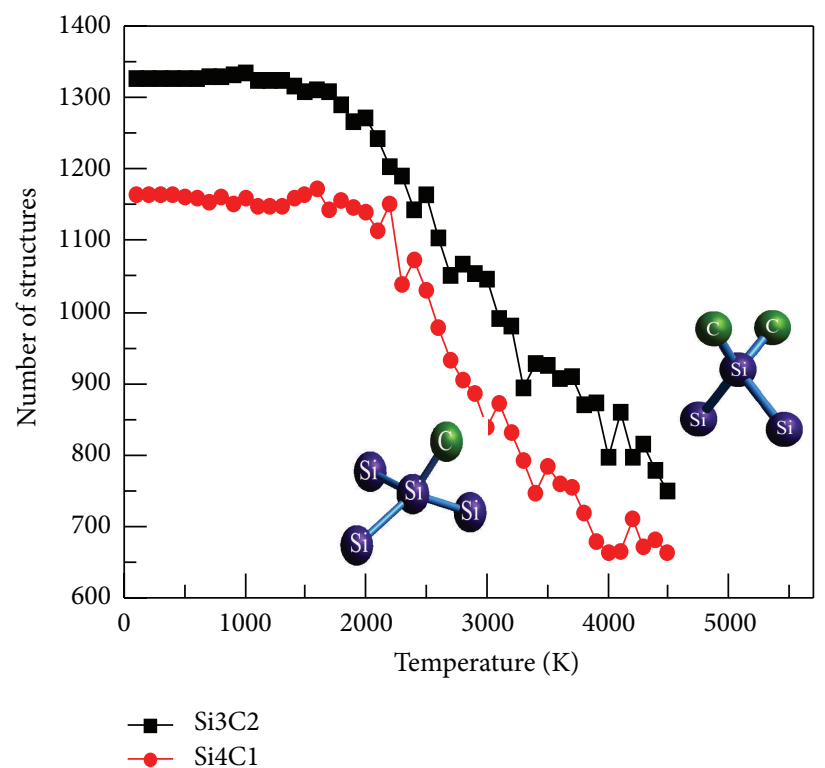

(c)

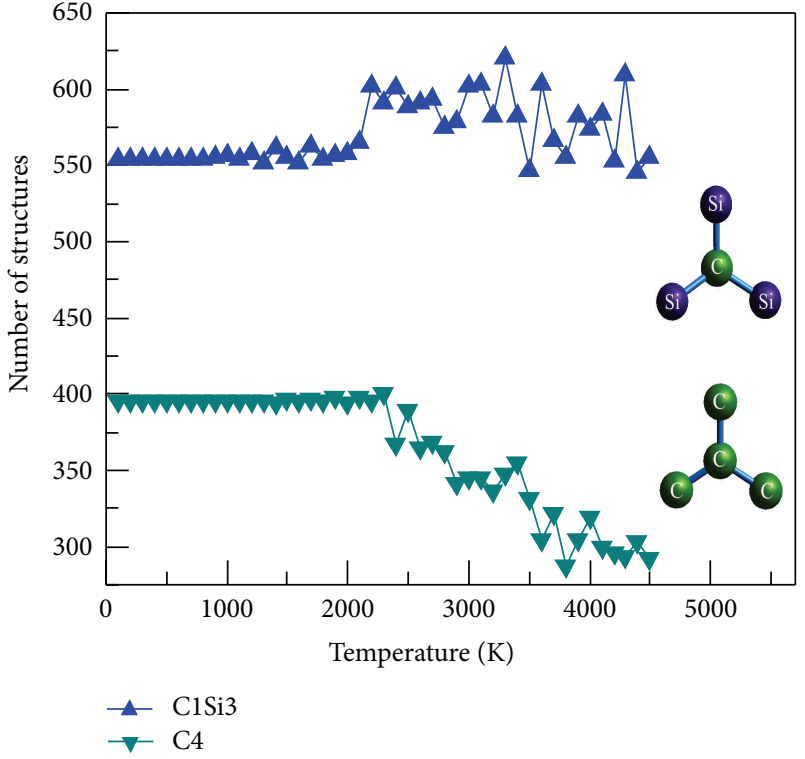

(b)

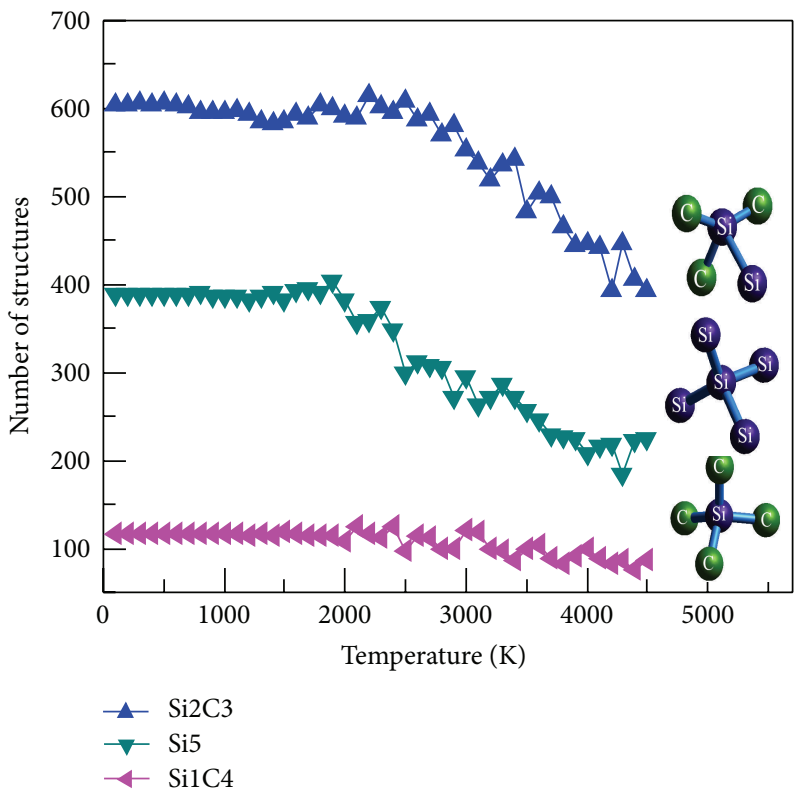

(d)

Figure 5: The number of main structures of $\mathrm{C}$ or $\mathrm{Si}$ as central atom with temperature decrease. ((a), (b) threefold coordination structures of $\mathrm{C}$ as central atom; (c), (d) fourfold coordination structures of Si as central atom).

structures connected by sharing the neighboring silicon atom and forming a short-range structure $\mathrm{Si}-\mathrm{Si}-\mathrm{Si}$. The distance of the two farthermost silicon atoms is $\sim 3.15 \AA$, which corresponds to the location of the second peak of $g_{\mathrm{Si}-\mathrm{Si}}(r)$ in Figure 1(c).

Figure 7 is the local area network-like amorphous structure at the end of the solidification of liquid $\mathrm{SiC}$, and the six typical structures above-mentioned are illustrated in Figure 7. Threefold carbon atoms are not the unsaturated bond or dangling bonds of a tetrahedral mesh but form a graphite-like structure (bond angle, $120^{\circ}$; bond length, $\sim 1.46 \AA$ ). Carbon atoms with numbers. 2647, 1502, 2929, and 3482 form a C-C-C-C short-range structure. Ultimately, no hexagonal graphite structure forms in the end because of the distorted connection. Five center silicon atoms with numbers. 5951, 5241, 5777, 7016, and 7055 of fourfold coordination structures connected individually by each center atom to form a diamond-like structure (bond angle, $109^{\circ}$; bond length, $\sim 2.35 \AA$ ). No diamond structure forms in the end because the coordination atoms of silicon deviate from 


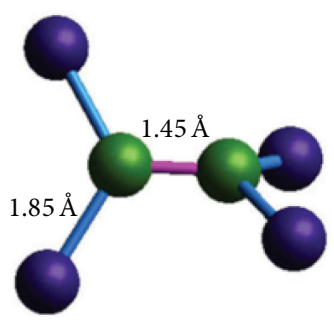

(a)

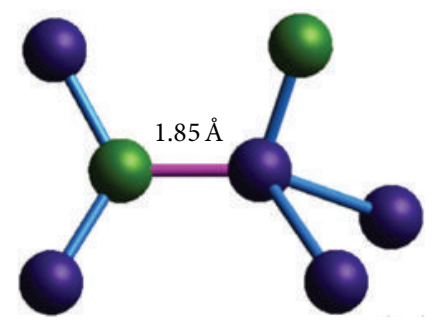

(b)

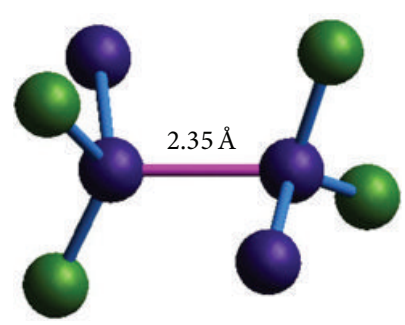

(c)

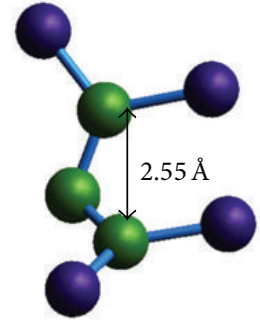

(d)

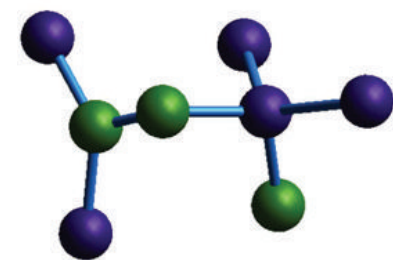

(e)

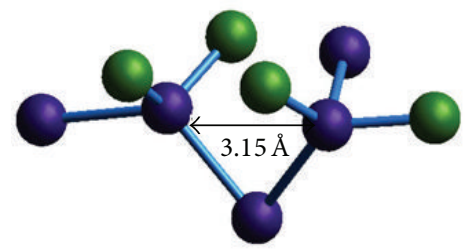

(f)

FiguRE 6: Six typical combination modes of carbon atoms and silicon atoms. ((a), (b), and (c) central atom is the neighbor atom of 3-3, 3-4, 4-4 coordination, (d), (e), and (f) sharing neighbor atom of 3-3, 3-4, 4-4 coordination).

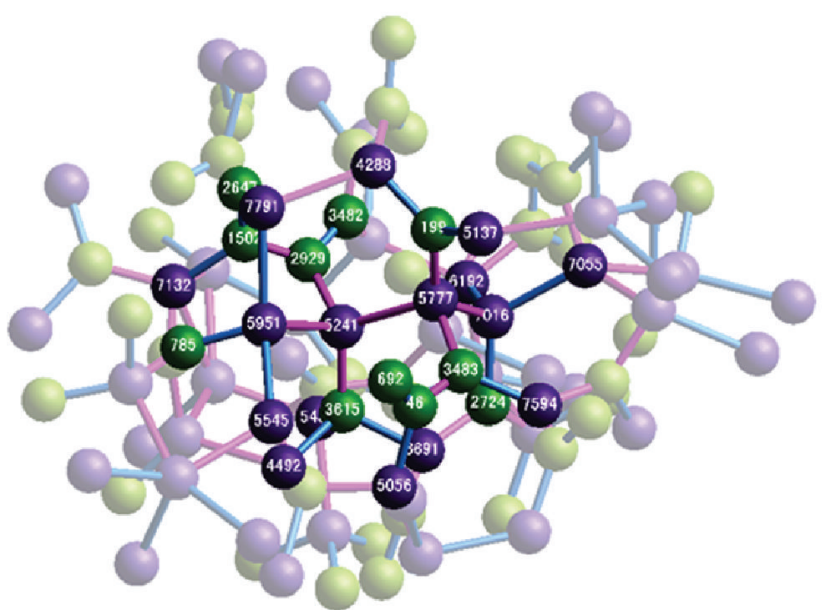

FIGURE 7: LAN amorphous structure at the end of solidification of SiC.

the crystal lattice positions of SiC. A random network amorphous structure is formed by connecting the threefold carbon atoms and fourfold silicon atoms at the end of solidification.

\section{Conclusions}

The rapid solidification of liquid $\mathrm{SiC}$ containing 8000 atoms is simulated with the Tersoff potential using MD. The microstructure properties of liquid and amorphous $\mathrm{SiC}$ are analyzed in detail by $\mathrm{RDF}, \mathrm{ADF}, \mathrm{CN}$, and visualization technology. Results show that with decreased temperature, the distance of $\mathrm{C}-\mathrm{C}$ and $\mathrm{Si}-\mathrm{Si}$ bonds gradually decreases, the $\mathrm{C}-\mathrm{Si}$ bond remains at $1.85 \AA$, and the SRO increases during solidification. Heteronuclear and homonuclear bonds coexist and no carbon or silicon atomic segregation occurs in the system. The average $\mathrm{CN}$ of $\mathrm{SiC}$ is $<4$ throughout the entire solidification process. Silicon atoms mainly form fourfold coordination structures with a tetrahedron bond angle of $109^{\circ}$, and carbon atoms mainly form threefold coordinationbased structure with a graphite bond angle of $120^{\circ}$ during solidification. The short-range structure is distorted because of the $\mathrm{C}-\mathrm{C}-\mathrm{C}$ connection. The coordination atoms of silicon deviate from the crystal lattice positions of SiC. Ultimately, no hexagonal ring of graphite and diamond structure forms. A random network amorphous structure is formed by connecting threefold coordination structures of $\mathrm{C}$ and fourfold coordination structures of $\mathrm{Si}$ at the end of solidification.

\section{Acknowledgments}

This work was supported by the National Natural Science Foundation of China (Grant no. 61264004), the Funds for 
International Sci-Tech Cooperation of Guizhou Province (Grant no. [2012]7004), the Special Funds for Construction of Sci-Tech Innovative Talents Team of Guizhou Province (Grant no. [2011]4002), Key Technologies Research and Development Project of Guizhou Province (Grant no. [2011]3015), the Special Fund for the Twelfth Five-YearMajor Sci-Tech Program of Education Department of Guizhou Province (Grant no. [2012]003), and Sci-Tech Program of Guiyang City of Guizhou Province of China under Grant no. [2012101] 2-16.

\section{References}

[1] B.-S. Lee, G. W. Burr, R. M. Shelby et al., "Observation of the role of subcritical nuclei in crystallization of a glassy solid," Science, vol. 326, no. 5955, pp. 980-984, 2009.

[2] X.-H. Yi, R.-S. Liu, Z.-A. Tian, Z.-Y. Hou, X. Wang, and Q.-Y. Zhou, "Simulation study of effect of cooling rate on evolution of microstructures during solidification of liquid metal Cu," Acta Physica Sinica, vol. 55, no. 10, pp. 5386-5393, 2006.

[3] Z.-Y. Hou, R.-S. Liu, X. Wang, Z.-A. Tian, Q.-Y. Zhou, and Z.H. Chen, "Simulation study of effects of initial melt temperature on microstructure of liquid metal $\mathrm{Na}$ during solidification processes," Acta Physica Sinica, vol. 56, no. 1, pp. 376-383, 2007.

[4] H.-T. Zhang, R.-S. Liu, Z.-Y. Hou, A.-L. Zhang, X.-Y. Chen, and S.-H. Du, "Simulation study for the effects of cooling rate on evolution of microstructures during solidification of liquid metal Ga,' Acta Physica Sinica, vol. 55, no. 5, pp. 2409-2417, 2006.

[5] Y. Lin, R.-S. Liu, Z.-A. Tian, Z.-Y. Hou, L.-L. Zhou, and Y.-B. Yu, "Effect of cooling rates on microstructures during solidification process of liquid metal Zn," Acta Physico Chimica Sinica, vol. 24, no. 2, pp. 250-256, 2008.

[6] Y. T. Yang, K. L. Ekinci, X. M. H. Huang et al., "Monocrystalline silicon carbide nanoelectromechanical systems," Applied Physics Letters, vol. 78, no. 2, pp. 162-164, 2001.

[7] M. Tang and S. Yip, "Atomistic simulation of thermomechanical properties of $\beta$-SiC," Physical Review B, vol. 52, no. 21, pp. 15150$15159,1995$.

[8] C. Koitzsch, D. Conrad, K. Scheerschmidt, and U. Gösele, "Empirical molecular dynamic study of $\mathrm{SiC}(0001)$ surface reconstructions and bonded interfaces," Journal of Applied Physics, vol. 88, no. 12, pp. 7104-7109, 2000.

[9] D. Stoltz, S. E. Stoltz, S. M. Widstrand, and L. S. O. Johansson, "Investigation of surface structure related features in the multiple-scattering simulations of photoelectron diffraction of 3C-SiC(001)-c $(4 \times 2)$," Physica B, vol. 395, no. 1-2, pp. 130-137, 2007.

[10] A. Chatterjee, A. Bhat, and K. Matocha, "Investigation of electrically active defects of silicon carbide using atomistic scale modeling and simulation," Physica B, vol. 401-402, pp. 81-84, 2007.

[11] N.-G. Zhou, T. Hong, and L. Zhou, "A comparative study between MEAM and Tersoff potentials on the characteristics of melting and solidification of carborundum," Acta Physica Sinica, vol. 61, no. 2, Article ID 028101, 2012.

[12] H. Pan and X. Si, "Molecular dynamics simulations of diameter dependence tensile behavior of silicon carbide nanotubes," Physica B, vol. 404, no. 12-13, pp. 1809-1812, 2009.
[13] Y. Zhang and H. Huang, "Stability of single-wall silicon carbide nanotubes-molecular dynamics simulations," Computational Materials Science, vol. 43, no. 4, pp. 664-669, 2008.

[14] Z. G. Wang, J. B. Li, F. Gao, and W. J. Weber, “Tensile and compressive mechanical behavior of twinned silicon carbide nanowires," Acta Materialia, vol. 58, no. 6, pp. 1963-1971, 2010.

[15] J. Wang, C. Lu, Q. Wang et al., "Influence of microstructures on mechanical behaviours of $\mathrm{SiC}$ nanowires: a molecular dynamics study," Nanotechnology, vol. 23, no. 2, Article ID 025703, pp. 110, 2012.

[16] W. C. Swope, H. C. Andersen, P. H. Berens, and K. R. Wilson, "A computer simulation method for the calculation of equilibrium constants for the formation of physical clusters of molecules: application to small water clusters," The Journal of Chemical Physics, vol. 76, no. 1, pp. 637-649, 1982.

[17] J. Tersoff, "Modeling solid-state chemistry: interatomic potentials for multicomponent systems," Physical Review B, vol. 39, no. 8, pp. 5566-5568, 1989.

[18] P. C. Kelires, "Short-range order and energetics of disordered silicon-carbon alloys," Physical Review B, vol. 46, no. 16, pp. 10048-10061, 1992.

[19] A. E. Kaloyeros, R. B. Rizk, and J. B. Woodhouse, "Extended xray-absorption and electron-energy-loss fine-structure studies of the local atomic structure of amorphous unhydrogenated and hydrogenated silicon carbide," Physical Review B, vol. 38, no. 18, pp. 13099-13106, 1988.

[20] S. Pascarelli, F. Boscherini, S. Mobilio, and F. Evangelisti, "Structure of hydrogenated amorphous silicon-carbon alloys as investigated by extended x-ray-absorption fine structure," Physical Review B, vol. 45, no. 4, pp. 1650-1654, 1992.

[21] S. L. Zhang, Simulation of melting and rapid solidification of silicon [dissertation for doctoral degree], Yanshan University, Hebei, China, 2010. 

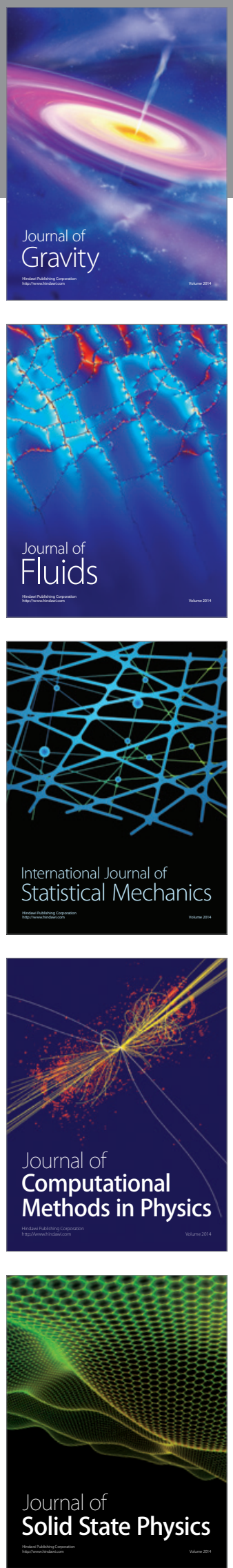

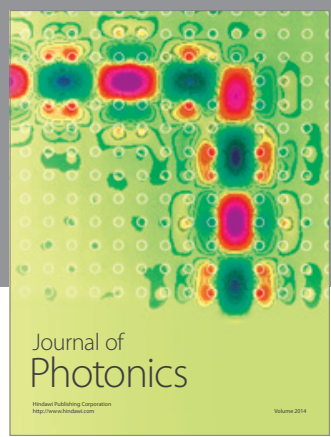

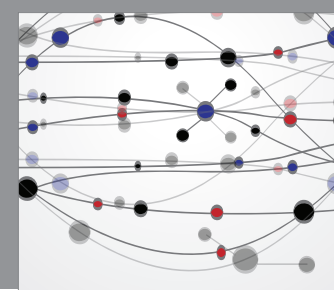

The Scientific World Journal

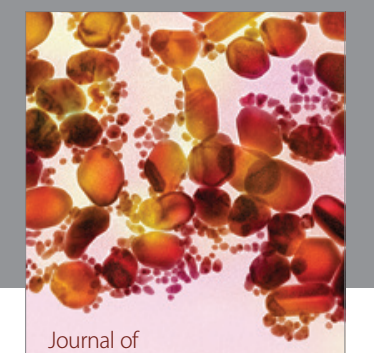

Soft Matter
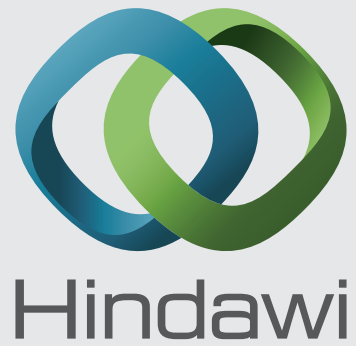

Submit your manuscripts at

http://www.hindawi.com
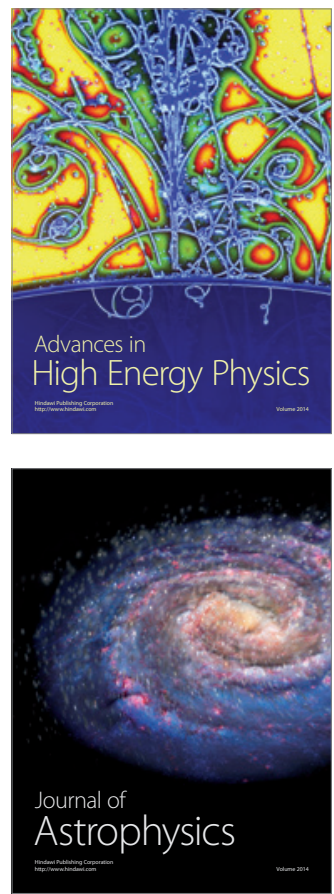
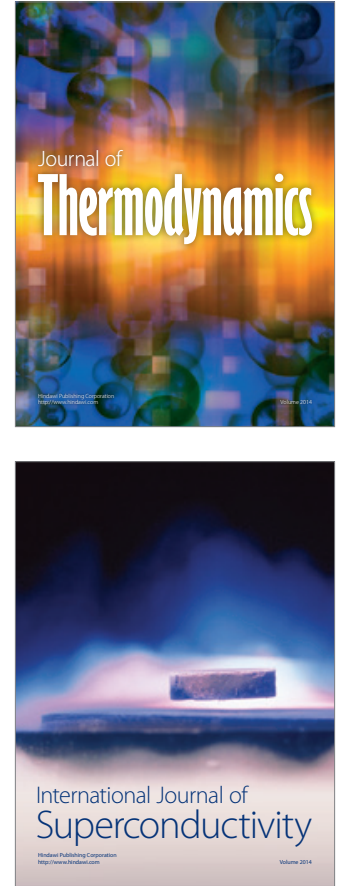
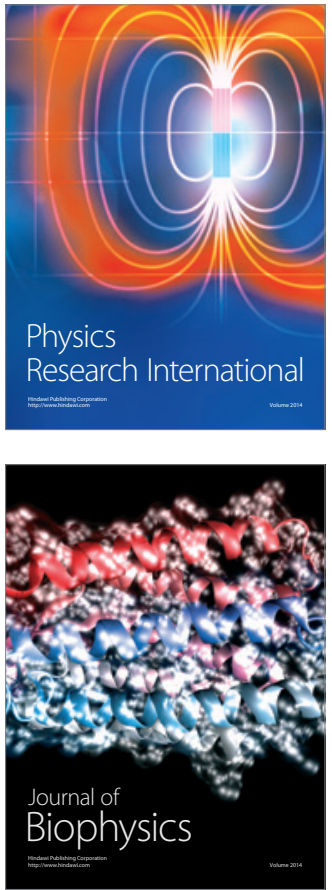
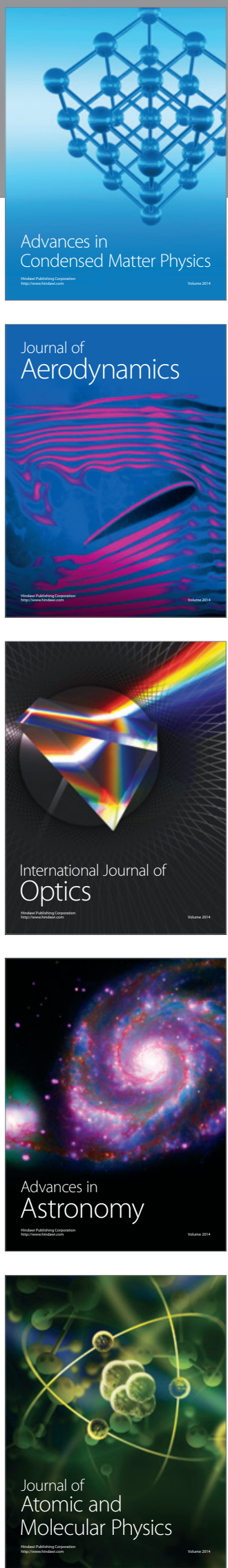